\title{
SÍNDROME DA ANGÚSTIA RESPIRATÓRIA AGUDA (SARA)
}

\author{
ACUTE RESPIRATORY DISTRESS SYNDROME (ARDS) \\ Paulo Antoniazzi ${ }^{1}$; Gerson Alves Pereira Júnior ${ }^{1}$; Flávio Marson ${ }^{1}$; Mario Abeid ${ }^{1}$; \\ Sérgio Baldisserotto ${ }^{2} \&$ Anibal Basile-Filho ${ }^{3}$
}

\begin{abstract}
${ }^{1}$ Médicos Intensivistas da UTI da Unidade de Emergência. ${ }^{2}$ Médico Residente da Disciplina de Terapia Intensiva. ${ }^{3}$ Docente e Chefe da Disciplina de Terapia Intensiva. Departamento de Cirurgia, Ortopedia e Traumatologia da Faculdade de Medicina de Ribeirão Preto da Universidade de São Paulo.

CorRespondências: Anibal Basile Filho - Disciplina de Terapia Intensiva, Departamento de Cirurgia, Ortopedia e Traumatologia da Faculdade de Medicina de Ribeirão Preto da Universidade de São Paulo - Hospital das Clínicas - 9 Andar - Campus Universitário CEP: 14048-900 - Ribeirão Preto - SP; Fone: (016) 633-0836 ou 602-2593. Email: abasile@.fmrp.usp.br
\end{abstract}

ANTONIAZZI $P$ et al. Síndrome da angústia respiratória aguda (SARA). Medicina, Ribeirão Preto, 31: 493-506, out./dez. 1998.

RESUMO: A síndrome da angústia respiratória aguda (SARA) é descrita como um quadro de lesão pulmonar aguda, associada a um edema pulmonar, agudo, não hidrostático e hipoxemia severa, acompanhado de altas taxas de mortalidade, entre 10 e $90 \%$ (média $=50 \%$ ), dependendo do fator etiológico.

Apesar de a SARA ter sido descrita há mais de trinta (30) anos, várias dúvidas ainda persistem quanto à sua definição, fisiopatologia e tratamento, em decorrência da complexidade e diversidade dos fatores envolvidos. O objetivo desse artigo de revisão é descrever a SARA nos seus aspectos clínicos, radiológicos e fisiopatológicos, abordando os fatores de risco, estratégias terapêuticas e as técnicas de assistência ventilatória, empregadas no seu tratamento.

UNITERMOS: Insuficiência Respiratória. Anoxemia. Síndrome do Desconforto Respiratório em Adultos.

\section{INTRODUÇÃO}

Em 1967, Ashbaugh et al. ${ }^{(1)}$ analisaram uma série de duzentos e setenta e dois (272) pacientes, submetidos à ventilação mecânica e identificaram doze (12) pacientes que evoluíram, agudamente, com taquipnéia, hipoxemia, diminuição da complacência do sistema respiratório e infiltrados pulmonares, difusos, na radiografia de tórax. Quatro anos mais tarde, esses mesmos autores denominaram esse conjunto de sinais e sintomas de Síndrome da Angústia Respiratória do Adulto (SARA) ${ }^{(2)}$. Existem várias definições para os critérios clínicos de SARA, ainda não aceitas uniformemente ${ }^{(3 / 8)}$. Originalmente, a maioria dessas definições incluiam três critérios clínicos básicos: a) hipoxemia (de intensidade variável); b) diminuição da complacência pulmonar; e c) infiltrados pulmonares na radiografia de tórax ${ }^{(6)}$. Com o aumento da disponibilidade e utilização da monitorização hemodinâmica invasiva (cateter de Swan-Ganz), nas Unidades de Terapia Intensiva, a SARA foi posteriormente classificada como um edema pulmonar, não cardiogênico, caracterizada pelo aumento da permeabilidade da membrana alvéolo-capilar, com extravasamento de células e proteínas para o interstício pulmonar. No início dos anos 80, nosso conhecimento sobre SARA aumentou, quando condições clínicas, específicas, que predispõem ao desenvolvimento da síndrome, foram publicadas $^{(4,5)}$. Através desses relatos importantes, dois novos critérios foram incluídos na definição de 
SARA: a) um fator clínico de risco; e b) pressão de enchimento ventricular, esquerda, nor$\mathrm{mal}^{(9 / 12)}$. Em 1988, Murray et al. ${ }^{(6)}$ propuseram um critério de pontuação ("score") da lesão pulmonar, no sentido de avaliar, definir e quantificar melhor a injúria pulmonar. Esse "score"foi amplamente utilizado em vários estudos clínicos ${ }^{(13 / 16)}$, e é baseado em quatro critérios, numa escala de 0 a $4^{(17)}$ (Tabela I). Em virtude das dificuldades em determinar-se a incidência da SARA, a heterogeinicidade das doenças de base, as suas várias definições e a não uniformidade da terapia, foi realizado, na Espanha, em 1992, "The American-European Consensus Conference on ARDS"(18), na tentativa de clarear e uniformizar as definições de SARA e lesão pulmonar aguda (LPA). Foi decidido que o termo a ser empregado para essa patologia complexa deveria ser mesmo aquele proposto inicialmente por Ashbaugh et al. ${ }^{(1)}$, porém, em vez do termo adulto, empregou-se o termo agudo, devido ao fato de a SARA não ser só limitada a adultos ${ }^{(1)}$, pois, naquela comunicação original, um (1) dos doze (12) pacientes relatados tinha onze (11) anos. Nessa conferência o "comitê de experts" recomendou que a lesão pulmonar aguda (LPA) fosse definida como uma síndrome de inflamação e um aumento da permeabilidade capilar, associada a uma série de alterações clínicas, radiológicas e fisiopatológicas. A sua associação com a sepse é muito frequiente, porém outras etiologias, como a aspiração pulmonar, a pneumonia primária ou os politraumatismos e, em menor escala, o curto-circuito ("by-pass") cardiopulmonar, as transfusões múltiplas, a embolia gordurosa, a pancreatite e outros fatores etiológicos também estão implicados. A SARA e a LPA têm um início agudo, com duração variável no período de instalação (pode durar de dias a semanas), presença de um fator de risco conhecido, e é caracterizada, principalmente, por hipoxemia persistente, refratária à terapia com oxigênio e infiltrados radiológicos, difusos. Doenças pulmonares crônicas, como fibrose intersticial pulmonar, sarcoidose e outras, devido à sua cronicidade, estão excluídas dessa definição. Os critérios recomendados para LPA e SARA podem ser visualizados no Tabela II.
Tabela I - Componentes do sistema de graduação (escore) da lesão pulmonar

\begin{tabular}{|ll|}
\hline & Valor \\
\hline Escore da radiografia de tórax & \\
\hline Nenhuma condensação alveolar & 0 \\
Condensação alveolar em um (1) quadrante & 1 \\
Condensação alveolar em dois (2) quadrantes & 2 \\
Condensação alveolar em três (3) quadrantes & 3 \\
Condensação alveolar em todos os quatro (4) & \\
quadrantes & 4 \\
Escore da hipoxemia & \\
\hline
\end{tabular}

$\mathrm{PaO}_{2} / \mathrm{F}_{1} \mathrm{O}_{2} \geq 300$
$\mathrm{PaO}_{2} / \mathrm{F}_{1} \mathrm{O}_{2}$ de $225-299$
$\mathrm{PaO}_{2} / \mathrm{F}_{1} \mathrm{O}_{2}$ de $175-224$
$\mathrm{PaO}_{2} / \mathrm{F}_{1} \mathrm{O}_{2}$ de $100-174$
$\mathrm{PaO}_{2} / \mathrm{F}_{1} \mathrm{O}_{2}<100$

Escore da complacência do sistema respiratório (quando ventilado) $\left(\mathrm{mL} / \mathrm{cmH}_{2} \mathrm{O}\right)$

$\begin{array}{ll}\geq 80 & 0 \\ 60-79 & 1 \\ 40-59 & 2 \\ 20-39 & 3 \\ \leq 20 & 4\end{array}$

Escore da pressão expiratória positiva final (quando ventilado) $\left(\mathrm{cmH}_{2} \mathrm{O}\right)$

\begin{tabular}{lc}
$\leq 5$ & 0 \\
$6-8$ & 1 \\
$9-11$ & 2 \\
$12-14$ & 3 \\
$\geq 15$ & 4 \\
$\begin{array}{l}\text { O valor final é obtido dividindo-se a soma total } \\
\text { pelo número de componentes usados }\end{array}$ \\
$\begin{array}{l}\text { Escore } \\
\text { Nenhuma lesão } \\
\text { Lesão leve a moderada } \\
\text { Lesão grave (SARA) }\end{array}$ \\
\hline
\end{tabular}




\section{Tabela II - Critérios diagnósticos de lesão pulmonar aguda (LPA) e da síndrome da angústia respiratória aguda (SARA). \\ LPA \\ 1. Início agudo. \\ 2. $\mathrm{PaO}_{2} / \mathrm{F}_{1} \mathrm{O}_{2}$ entre 200 e $300 \mathrm{mmHg}$ (apesar do uso de pressão positiva na fase final da expiração-PEEP). \\ 3. Radiografia de tórax, mostrando infiltrados alvéolo- intersticiais, micro e/ ou macronodulares, bilaterais e assimétricos. \\ 4. Pressão capilar pulmonar $<18 \mathrm{mmHg}$ ou ausência de evidências clínicas de hipertensão atrial esquerda \\ SARA \\ 1. Mesmos critérios descritos para LPA \\ 2. $\mathrm{PaO}_{2} / \mathrm{F}_{1} \mathrm{O}_{2}<200 \mathrm{mmHg}$ (apesar do uso de PEEP).}

\section{ETIOLOGIA}

A SARA é um processo de edema pulmonar, agudo, não hidrostático ou não cardiogênico, acompanhado de uma hipoxemia persistente, associado a uma ampla lista de situações que predispõem ao seu desenvolvimento, e que pode atingir um índice de mortalidade, dependendo do fator etiológico envolvido, de $10 \%$ a $90 \%{ }^{(18,19,20)}$. Calcula-se que a incidência de SARA, nos Estados Unidos, está em torno de cento e cinqüienta mil (150.000) casos/ano. A maior causa de SARA (43\% dos casos) ocorre nos pacientes com síndrome séptica ${ }^{(11)}$. A probabilidade de um paciente desenvolver a síndrome aumenta a medida que um ou mais fatores de risco estão presentes. Por esta razão, é de fundamental importância identificar o paciente com o risco de desenvolver a síndrome, pois, quanto mais precocemente se intervém na causa básica, melhor será o prognóstico. Os fatores de risco mais comumente relacionados à SARA são os seguintes:

\subsection{Injúria direta}

- Aspiração

- Infecção pulmonar, difusa (bacteriana, viral, pneumocystis e outros)

- Afogamento

- Inalação tóxica

- Contusão pulmonar

- Embolia gordurosa

- Toxicidade pelo oxigênio

\subsection{Injúria indireta}

- Síndrome séptica

- Politraumatizado

- Politransfusão

- Choque

- Grandes queimados

- Pancreatite

- "By-pass" cardiopulmonar

- Intoxicação exógena

- Coagulação intravascular, disseminada

- Excesso de fluidos

\section{PATOGÊNESE}

A lesão pulmonar aguda, que origina a SARA, caracteriza-se pela injúria do endotélio e epitélio pulmonar e, consequientemente, com a lesão da membrana alvéolo-capilar. Existe uma série de mediadores circulantes, humorais ou celulares, que são imputados como causadores e perpetuadores da síndrome, mas, em geral, sua patogênese consiste em duas vias: a) os efeitos diretos de uma lesão nas células pulmonares; e b) uma resposta inflamatória, sistêmica, aguda, que pode incluir componentes celulares e humorais. A resposta celular envolve neutrófilos, macrófagos/monócitos e linfócitos, os quais têm um papel importante no processo que inclui a adesão, a quimiotaxia e a ativação dos leucócitos.

Essa interação intercelular é feita por compostos denominados de moléculas de adesão (adesinas, integrinas e selectinas), que podem regular a expressão das células endoteliais e inflamatórias, tendo um papel fundamental na perpetuação das respostas celulares, inflamatórias, que culminam com a disfunção de múltiplos órgãos ${ }^{(21)}$. Os eventos envolvidos na resposta inflamatória sistêmica incluem alterações plasmáticas (sistema complemento, coagulação/fibrinólise e cininas), mediadores gerados pelas células (citocinas, mediadores lipídicos, oxidantes, proteases, óxido nítrico e neuropeptídeos) e indução de síntese protéica, um estágio precoce na produção de citocinas (Figura 1). Esses fatores são considerados responsáveis pela injúria celular e alterações no surfactante, com distúrbio da relação ventilação/perfusão, resultando em hipoxemia. As endotoxinas exercem efeito na ativação de sistemas enzimáticos do plasma, complemento e liberação do fator de Von Willenbrand, propiciando fenômenos trombóticos e hipercoagulabilidade, além de ter, também, efeito lesivo, direto no endotélio dos vasos pulmonares ${ }^{(22)}$. 


\title{
Endotoxina
}

\author{
$\downarrow$ \\ Neutrófilos e Macrófagos \\ Quimiotaxia e Ativação
}

$\downarrow$

Liberação de Mediadores

TNF*, Prostaglandinas, Radicais de Oxigênio, PAF**

Leucotrienos, IL-t**

Lesão celular endotelial, Edema intersticial, Exudação de proteínas

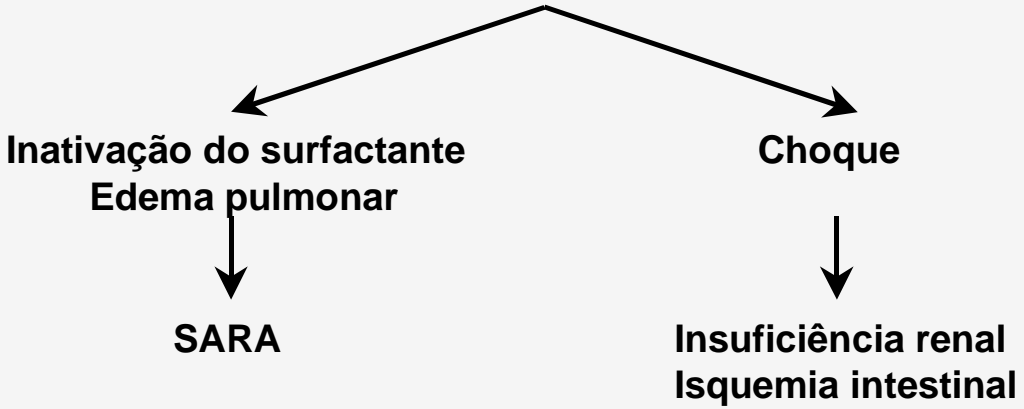

Figura 1 - Esquema proposto para a patogênese da Síndrome da Angústia Respiratória Aguda (SARA) e da Síndrome de Disfunção de Múltiplos Órgãos (SDMO).

* TNF = Fator de necrose tumoral. ${ }^{* *} \mathrm{PAF}=$ Fator de ativação plaquetária. ${ }^{* * *} \mathrm{IL}-1=$ Interleucina 1

Deve ser ressaltado que a mais importante das citocinas liberadas na sepse é o fator de necrose tumoral (TNF) ou caquexina, que, além de possuir um efeito citotóxico, direto, no endotélio, estimula, também, a produção de outras citocinas como as interleucinas. Essas substâncias interagem com células endoteliais, aumentando a atividade pró-coagulante e a produção do inibidor do plasminogênio, facilitando a coagulação e promovendo a ativação de neutrófilos, monócitos e linfócitos que, por sua vez, liberam ânions superóxidos e outros radicais livres que participam na lesão do tecido endotelial, desempenhando, portanto, importante papel na gênese da SARA ${ }^{(23)}$. Os derivados do ácido araquidônico (prostaglandinas, prostaciclinas e tromboxano) também estão implicados na patogênese da SARA, devido ao seu potente efeito no aumento da permeabilidade vascular.

\section{PATOLOGIA PULMONAR NA SARA}

As anormalidades patológicas do pulmão, na SARA, originam-se da lesão grave da unidade alvéolo-capilar. O extravazamento do líquido intravascular predomina no início e, à medida que o processo evolui, o edema é substituído pela necrose celular, hiperplasia epitelial, inflamação e fibrose, caracterizando uma lesão alveolar, difusa ${ }^{(24)}$. A SARA pode ser dividida em três fases, sendo cada fase variável de acordo com o tempo e a evolução clínica da doença: a "fase exsudativa", de edema e hemorragia, a "fase proliferativa", de organização e reparação, e a "fase de fibrose".

\subsection{Fase exsudativa}

Estende-se, geralmente, durante a primeira semana após o início da insuficiência respiratória, e as alterações vistas na microscopia óptica são a 
congestão capilar, o edema alvéolo-intersticial e a hemorragia intra-alveolar ${ }^{(25)}$. Nessa fase, há uma necrose extensa do epitélio alveolar, com perda da barreira epitelial, alveolar e livre passagem do líquido intersticial para o espaço alveolar, caracterizando, assim, o edema pulmonar não hidrostático.

\subsection{Fase proliferativa}

Esse é o estágio de organização dos exsudatos intra-alveolares e intersticiais, observados na fase agu$\mathrm{da}^{(26)}$. Os pneumócitos do tipo II proliferam-se nos septos alveolares a partir do terceiro dia do início da SARA e a fibrose é evidenciada em torno do décimo dia. Os fibroblastos convertem o exsudato em tecido de granulação celular e, posteriormente, pela deposição de colágeno, em tecido fibroso denso. Após a lesão da unidade alvéolo-capilar, as paredes alveolares colapsam, e tornam-se vedadas pela deposição de fibrina. Anormalidades estruturais e funcionais do surfactante também contribuem para o colapso alveolar, na SARA ${ }^{(27)}$.

\subsection{Fase de fibrose pulmonar}

Nos pacientes sob ventilação mecânica, que sobrevivem por três (3) ou quatro (4) semanas do início da SARA, o pulmão é totalmente remodelado por tecido rico em fibras de colágeno ${ }^{(28)}$. Além do colágeno, há um aumento de elastina, fibras musculares lisas e glicoproteínas com comprometimento de todo o sistema alvéolo-capilar, envolvido nas trocas gasosas, levando à hipoxemia grave refratária e hipertensão arterial pulmonar, responsáveis pela fase terminal da SARA $^{(29)}$. É preciso enfatizar ainda que, além das alterações citadas acima, o comprometimento da vascularização pulmonar é um aspecto crucial na SARA, que se estende desde os estágios iniciais até as fases terminais. Essas lesões incluem alterações trombóticas, fibroproliferativas e obstrutivas que, a exemplo das lesões parenquimatosas, também se correlacionam com a fase evolutiva da lesão alveolar difusa.

\section{ALTERAÇÕES CLÍNICAS E FUNCIO- NAIS DA SARA}

É importante salientar que o quadro clínico inicial pode caracterizar-se apenas por taquipnéia e alcalose respiratória, sem hipoxemia ou alteração radiológica. A evolução do quadro clínico para insuficiência respiratória franca pode ser súbita. $O$ principal sintoma da SARA é a dispnéia. Os sinais clínicos são reflexos da hipoxemia e do edema pulmonar, e inclu- em taquicardia, taquipnéia com batimentos das asas do nariz e cianose. Roncos e estertores pulmonares bilaterais e difusos podem estar presentes. A radiografia de tórax mostra infiltrados pulmonares bilaterais e difusos, na maioria dos casos assimétricos, sem evidência de cardiomegalia (Figura 2). Dentre as alterações funcionais mais importantes, destacam-se a diminuição da complacência pulmonar (variação de volume pulmonar/pressão), devido ao edema pulmonar, aos infiltrados inflamatórios e às atelectasias, ou fibrose, que diminuem a distensibilidade do pulmão. A capacidade residual funcional, que é a quantidade de ar que permanece nos pulmões após a expiração normal, está diminuída (alteração importante na SARA) devido às atelectasias e à exsudação de líquido para dentro dos espaços alveolares. Outro distúrbio característico da síndrome é o desequilíbrio da relação ventilação/perfusão, onde existem áreas perfundidas e não ventiladas, denominadas de "shunt pulmonar". Na SARA, o "shunt pulmonar" é o principal mecanismo gerador de hipoxemia e suas principais causas incluem: edema alveolar, trombos na microcirculação, adesividade celular e microatelectasias. Quando o grau de "shunt" é muito elevado, a hipoxemia é refratária à administração de oxigênio, mesmo em altas concentrações.

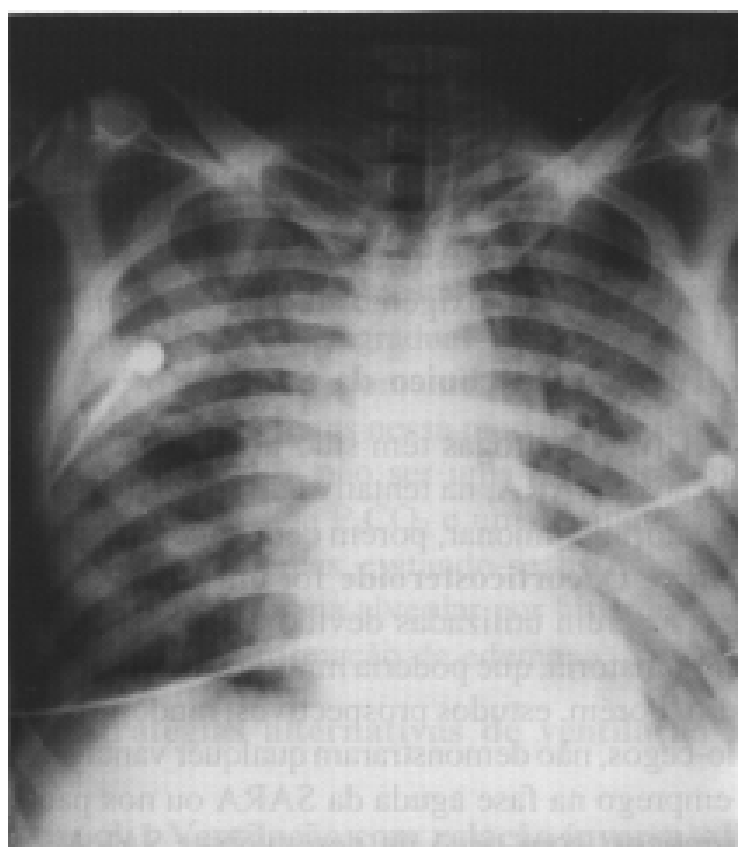

Figura 2 - Radiografia de tórax de um paciente com SARA, na fase exsudativa. Notar a presença de um infiltrado alvéolo-intersticial, bilateral e assimétrico. 


\section{TRATAMENTO}

O manuseio e o tratamento de pacientes com Síndrome de Angústia Respiratória Aguda (SARA) é um constante desafio para os médicos intensivistas que atuam nas unidades de terapia intensiva. Apesar dos importantes avanços tecnológicos nas últimas décadas, a mortalidade na SARA permanece elevada desde a descrição inicial da síndrome ${ }^{(1,30)}$. Na abordagem terapêutica da SARA, é de fundamental importância identificar o agente etiológico, pois, com a eliminação da causa, é possível interromper a história natural da doença. É também fundamental manter um consumo adequado de $\mathrm{O}_{2}$ pelos tecidos, pois sabe-se que a respiração celular depende, de um lado, do fornecimento de oxigênio aos tecidos periféricos e, de outro, da capacidade dos tecidos em extrair o $\mathrm{O}_{2}$ ofertado $^{(31)}$. Estes dados podem ser monitorizados, de maneira não invasiva, à beira do leito, através da calorimetria indireta. Porém, este método é pouco disponível em nosso meio, sendo que esses dados podem ser calculados através dos parâmetros obtidos com o catéter de Swan-Ganz. Existe um distúrbio generalizado da microcirculação na SARA, onde o pulmão apresenta uma alteração da relação ventilação/perfusão ( $\left.\mathrm{V}_{\mathrm{A}} / \mathrm{Q}\right)$, enquanto que, na periferia, ocorre distúrbio da relação oferta/consumo de oxigênio $\left(\mathrm{DO}_{2} / \mathrm{VO}_{2}\right)^{(32,33)}$. O distúrbio da microcirculação sistêmica pode estar relacionado aos mesmos fatores que lesam o leito capilar pulmonar, ou seja: edema intersticial, lesão endotelial, formação de microtrombos e trombos leucocitários, liberação do tromboxano $\mathrm{A}_{2}$ e outros mediadores e aumento da permeabilidade capilar. Esses fenômenos ocasionam obstáculos na difusão do oxigênio até a mitocôndria ${ }^{(34,35)}$.

\subsection{Tratamento clínico da SARA}

Diversas drogas têm sido utilizadas durante a evolução da SARA, na tentativa de reverter o quadro inflamatório pulmonar, porém com pouco sucesso terapêutico. O corticosteróide foi uma das primeiras drogas a serem utilizadas devido a sua potente ação antiinflamatória, que poderia minimizar ou bloquear o quadro. Porém, estudos prospectivos, randomizados e duplo-cegos, não demonstraram qualquer vantagem ao seu emprego na fase aguda da SARA ou nos pacientes sépticos, com risco de desenvolver SARA ${ }^{(36 / 40)}$. No entanto, algumas experiências têm mostrado que, quando o quadro pulmonar está evoluindo para uma fibrose extensa, os corticosteróides podem ter algum efeito protetor, principalmente se os quadros infeccio- sos estiverem afastados ${ }^{(41,42,43)}$. Apesar das controvérsias, somente em casos selecionados, como por exemplo, na fase precoce do paciente com embolia gordurosa, recomendamos, em nosso Serviço, a administração parenteral em "bolus" de 2 a $3 \mathrm{mg} / \mathrm{kg}$ de metilprednisolona, durante os três (3) primeiros dias de evolução da SARA.

O uso de surfactante sintético, via aerossol, pode ser eficaz em crianças com quadro de membrana hialina, contudo foi mostrado, num estudo multicêntrico, que não houve benefício com essa modalidade terapêutica na SARA ${ }^{(44)}$.

Outras drogas, como a prostaglandina $\mathrm{E}_{1}$, o ibuprofeno, a pentoxifilina, a $\mathrm{N}$-acetilcisteína e as antiendotoxinas têm sido empregadas experimentalmente, na SARA ${ }^{(45,46,47)}$, porém sem qualquer resultado satisfatório na redução da mortalidade, causada principalmente por: a) sepse associada com disfunção de múltiplos órgãos (tardia); e b) insuficiência respiratória progressiva.

\subsection{Ventilação mecânica na SARA}

Independente da etiologia, quase todos os pacientes que apresentam SARA necessitam de suporte ventilatório. A ventilação mecânica tem como finalidade propiciar aos pulmões o tempo necessário para se recuperarem da injúria aguda, onde os principais objetivos do tratamento ventilatório são: a) manter a troca gasosa e b) evitar as lesões pulmonares, associadas ao suporte ventilatório, ou seja, barotrauma, a toxicidade pelo oxigênio e ao que chamamos, nos dias de hoje, de "volutrauma". O termo volutrauma é um fenômeno causado por repetidas distensões e pressurizações do tórax, que podem causar uma indetectável cascata de reações inflamatórias no parênquima pulmonar, ocasionando um dano alveolar difuso, aumento da permeabilidade vascular e infiltrados inflamatórios, induzidos pela ventilação mecânica ${ }^{(48,49,50)}$. Esse efeito tem uma conotação clínica, diferente do termo barotrauma, muito usado na prática clínica diária ${ }^{(51)}$. A lesão pulmonar, produzida pela ventilação mecânica, é um fenômeno muito mais volume-dependente do que pressão-dependente, e isso ocorre em função do aumento da pressão transpulmonar e não da pressão alveolar.

$\mathrm{Na}$ atualidade, não existem estudos clínicos, na literatura, que comprovem a superioridade de um ou outro método ventilatório na SARA. Gattinoni et al., em 1986, e Hickling et al., em $1990^{(52,53)}$, associaram o tipo de suporte ventilatório, a morbidade/mortalidade e índices prognósticos (APACHE II), no sentido de compararem a sobrevida dos pacientes estudados. 
Esses autores concluíram não haver diferença entre a modalidade ventilatória, empregada e a redução da mortalidade. Todavia, alguns estudos têm mostrado superioridade de algumas técnicas ventilatórias sobre outras, principalmente em situações extremas. Como exemplo, a PC-IRV (ventilação com relação inversa e pressão controlada) ${ }^{(54,55)}$, o uso da posição prona ${ }^{(56)}$, a ventilação pulmonar independente ${ }^{(57)} \mathrm{e}$ a insuflação endotraqueal de gás ${ }^{(58)}$ são algumas modalidades alternativas, empregadas na SARA.

Baseados nesses relatos, e por não haver uma concordância a respeito da melhor estratégia ventilatória, deve-se optar pelo método com que a equipe médica estiver mais familiarizada a utilizar, e que esse promova uma oxigenação sangüínea e ventilação alveolar, adequadas. No entanto, qualquer que seja a modalidade ventilatória empregada, devem ser observados os seguintes pontos:

- manter uma $\mathrm{S}_{\mathrm{a}} \mathrm{O}_{2}>91 \%$;

- a pressão de platô (PPLAT), que é a pressão nas vias aéreas ao final da pausa inspiratória, não deve ultrapassar $35 \mathrm{cmH}_{2} \mathrm{O}$, podendo ser utilizado um volume corrente $\left(\mathrm{V}_{\mathrm{T}}\right)$ de até $5 \mathrm{~mL} / \mathrm{kg}$, ou menos, para atingir tal objetivo;

- liberar a $\mathrm{P}_{\mathrm{a}} \mathrm{CO}_{2}$ (hipercapnia permissiva) devido à limitação da PPLAT, evitando aumento da pressão intracraniana ou queda do $\mathrm{pH}$, utilizando reduções graduais do volume corrente;

- utilizar sempre pressão positiva no final da expiração (PEEP), com estrita vigilância sobre os seus efeitos colaterais; possível;

- menor fração inspirada de oxigênio $\left(\mathrm{F}_{\mathrm{I}} \mathrm{O}_{2}\right)$

- reduzir o consumo de oxigênio através da sedação/paralisia, e controle da temperatura;

- otimizar a oferta de $\mathrm{O}_{2}\left(\mathrm{DO}_{2}\right)$ para os tecidos, através do débito cardíaco e da concentração de hemoglobina;

- controle do fator etiológico, sepse e do edema pulmonar.

Desde os anos 60, a filosofia de tratamento dos pacientes com SARA é evitar ou desfazer as áreas de atelectasias, responsáveis pela hipoxemia, e impedir o colabamento dos alvéolos durante o ciclo expiratório, cujo objetivo final é produzir valores normais dos gases sangüíneos. Para tal propósito, utilizavam-se altos volumes correntes e altas freqüências respiratórias (FR), aumentando-se, assim, o volume minuto (VM). A fim de se evitarem as complicações da SARA (microatelectasias com distúrbio ventilação/perfusão), o uso de volume corrente $\left(\mathrm{V}_{\mathrm{T}}\right)$ entre 10 e $15 \mathrm{~mL} / \mathrm{kg}$ parecia, desde aquela época, ser a solução apropriada, não importando o nível de pressão gerado nas vias aéreas. Os objetivos dessa estratégia ventilatória eram produzir uma oxigenação adequada $\left(\mathrm{S}_{\mathrm{a}} \mathrm{O}_{2}>91 \%\right)$ e a remoção do $\mathrm{CO}_{2}$. A esse tipo de assistência ventilatória deu-se o nome de ventilação mecânica, convencional. Portanto, nesse tipo de ventilação, utilizam-se volumes correntes entre $10-15 \mathrm{~mL} / \mathrm{kg}$, com fluxo inspiratório de quarenta (40) a sessenta (60) litros/min, buscando-se uma relação insp/exp de 1:2, com forma de onda de fluxo, quadrada devido a menor pressão média, gerada nas vias aéreas, do que a forma descendente, e com menos efeitos hemodinâmicos ${ }^{(59)}$. A frequiência respiratória (FR) é comandada pelo paciente, no modo assistido, ou estipulada entre doze (12) a trinta (30) ciclos/min, no modo controlado, a fim de se obterem níveis de $\mathrm{P}_{\mathrm{a}} \mathrm{CO}_{2}$ entre 28 e $35 \mathrm{mmHg}$. FR maiores podem ocasionar auto $\mathrm{PEEP}^{(60)}$. $\mathrm{A} \mathrm{F}_{\mathrm{I}} \mathrm{O}_{2}$ deve ser a menor possível para proporcionar uma $\mathrm{P}_{\mathrm{a}} \mathrm{O}_{2}$ entre 60 e $100 \mathrm{mmHg}$, e, quando se fizer necessária uma $\mathrm{F}_{\mathrm{I}} \mathrm{O}_{2}>0,5$ para alcançar esses valores, utiliza-se a pressão positiva no final da expiração (PEEP), com a finalidade de manter os alvéolos abertos e melhorar a oxigenação. Parece haver um consenso geral de que o valor total de PEEP para impedir o colapso dos alvéolos, no ciclo respiratório, situa-se entre 7 e $15 \mathrm{mmHg}$. Os incrementos do PEEP devem ser feitos de forma gradual (3 a $5 \mathrm{cmH}_{2} \mathrm{O}$ ), com medidas de seus efeitos após 15 a 20 min, principalmente sobre o sistema cardiovascular. Deve ser indicada monitorização hemodinâmica, com cateter de Swan-Ganz sempre que os níveis de PEEP excedam $10 \mathrm{cmH}_{2} \mathrm{O}$. Da mesma forma, a retirada da PEEP também deve ser gradual.

Até o presente momento, poucas alterações substanciais foram feitas nessa modalidade de ventilação mecânica ${ }^{(61)}$, a não ser uma tolerância maior para com os níveis da $\mathrm{P}_{\mathrm{a}} \mathrm{CO}_{2}$ e uma diminuição nas pressões das vias aéreas, evitando-se, assim, uma lesão direta da membrana alveolar por hiperdistensão, com conseqüente formação de edema.

\subsection{Estratégias alternativas de ventilação me- cânica}

\subsubsection{Ventilação com relação inversa - IRV (Inverse Ratio Ventilation)}

A ventilação com relação inversa é uma abordagem ventilatória na qual se prolonga a inspiração e encurta-se o tempo expiratório, de forma que a rela- 
ção I/E seja superior a 1. Essa técnica permite utilizarem-se baixos níveis de pressão de distensão alveolar, ou seja, recrutar ao máximo os alvéolos com pressões de enchimento menores. A elevação sustentada de pressão, nas vias aéreas, parece recrutar alvéolos colabados mais eficazmente do que com aumentos transitórios da mesma. A relação insp/exp pode ser invertida através de dois métodos :

a) pressão controlada (PC-IRV), através do ajuste do tempo inspiratório;

b) volume controlado (VC-IRV), onde existe a possibilidade de se diminuir o fluxo inspiratório, para alcançar o tempo inspiratório desejado e introduzir a pausa inspiratória.

A ventilação com a relação insp/exp invertida é mais utilizada no modo pressão, controlado do que no modo volume, controlado. Na PC-IRV, determinase o nível de pressão, a frequiência respiratória e o tempo inspiratório, para obter-se um volume corrente desejado, sendo que esse volume corrente depende da resistência e complacência da árvore respiratória e dos ajustes na pressão determinada, freqüência respiratória e tempo inspiratório. Um aspecto importante da inversão da relação I/E é a necessidade de sedação profunda do paciente para sua tolerância com esse método. Essa modalidade ventilatória pode também ocasionar o fenômeno de auto PEEP, com queda do débito cardíaco. Geralmente, a relação I/E de 2:1, em pacientes hemodinamicamente estáveis, não altera o rendimento cardíaco. Embora o uso dessa modalidade venha aumentando nos pacientes com SARA, não há relatos que mostrem aumento na sobrevivência dos pacientes ventilados com esse método, porém, acredita-se que a inversão da relação I/E deva ser introduzida quando a ventilação convencional não produzir uma oxigenação adequada, com níveis aceitáveis de PEEP e $\mathrm{F}_{\mathrm{I}} \mathrm{O}_{2}{ }^{(54)}$, ou quando se deseja minimizar o risco de lesão pulmonar, produzida por altos níveis de pressão nas vias aéreas.

\subsubsection{Hipercapnia permissiva}

A hipercapnia permissiva consiste na limitação proposital do suporte ventilatório $\left(\mathrm{V}_{\mathrm{T}}\right.$ baixo) para evitar a hiperdistensão alveolar, permitindo-se níveis de $\mathrm{P}_{\mathrm{a}} \mathrm{CO}_{2}$ acima de $50 \mathrm{mmHg}^{(62,63)}$. Com o intuito de se manter uma normocapnia na SARA, através da ventilação mecânica, convencional, essa modalidade pode agravar ou ocasionar injúria da membrana alveolar. Através de estudos efetuados com tomografia computadorizada, ficou demonstrado que somente algu- mas regiões pulmonares estão disponíveis para ventilação, tendo sido esse fenômeno denominado de "baby lung", isto é, a área pulmonar efetiva para receber o volume corrente, liberado pelo ventilador (no pulmão de um adulto com SARA), pode ser equivalente à área do pulmão de uma criança. Assim, a aplicação de $\mathrm{V}_{\mathrm{T}}>10 \mathrm{~mL} / \mathrm{kg}$ pode resultar em desvio desse volume para regiões de melhor complacência, que pode ser demasiado, e levar ao aparecimento da lesão pulmonar. Por isso, alguns autores ${ }^{(53,64)}$ têm utilizado esse método de ventilação, com limitação de pressão nas vias aéreas ( $\mathrm{PI}<40 \mathrm{cmH}_{2} \mathrm{O}$ ), baixo volume corrente $\left(\mathrm{V}_{\mathrm{T}}\right.$ até $7 \mathrm{~mL} / \mathrm{kg}$ ) e permitindo hipercapnia, visando a proteção do pulmão já lesado pela SARA, ou seja, a ventilação mecânica para objetivar o "repouso pulmonar". A eleveção aguda da $\mathrm{P}_{\mathrm{a}} \mathrm{CO}_{2}$ ocasiona alterações do $\mathrm{pH}$ intracelular, levando a disfunção de sistema nervoso central, hipertensão intracraniana, fraqueza muscular e depressão cardiovascular. Todavia, quando corrigimos as variações do $\mathrm{pH}$ com bicarbonato e reduzimos de forma gradual o volume corrente, os efeitos indesejáveis da queda do $\mathrm{pH}$ podem ser evitados. Com o passar de alguns dias, ocorre retenção de bicarbonato pelos rins como mecanismo de compensação.

Trabalhos recentes ${ }^{(55,65,66)}$ têm mostrado que essa abordagem ventilatória, apesar dos níveis elevados da $\mathrm{P}_{\mathrm{a}} \mathrm{CO}_{2}$ (acima de $55 \mathrm{mmHg}$ ) e suas repercussões negativas, possui influência direta na evolução dos pacientes com SARA. Nota-se uma queda na mortalidade , maior probabilidade de sucesso do desmame e melhora da função pulmonar e da mecânica respiratória, vindo a ser um método promissor .

\subsubsection{Ventilação de alta freqëncia (HFJV High Frequency Jet Ventilation)}

A aplicação da ventilação de alta frequiência, no tratamento da SARA, baseia-se na possibilidade da HFJV conseguir oxigenar esses pacientes com frequiências ventilatórias maiores (acima de 60 ciclos $/ \mathrm{min}$ ) e pequenos volumes correntes, evitando-se lesões pulmonares, causadas por pressões elevadas, e os efeitos negativos da ventilação convencional no aparelho cardiocirculatório, diminuindo, também, os riscos de barotrauma. Apesar do otimismo causado pelo fato de que a ventilação de alta freqüência diminui as alterações hemodinâmicas, quando comparada com a ventilação mecânica, convencional, os trabalhos realizados não demonstraram diferenças significativas na sobrevivência dos pacientes ${ }^{(67)}$. No momento, a HFJV 
é empregada principalmente nos casos de insuficiência respiratória, associada à fístulas broncopleurais.

\subsubsection{Insuflação traqueal de gás (TGI - Tracheal Gas Insuflation)}

Esse método consiste na insuflação de ar através de cateteres localizados próximo à carina, com a finalidade de melhorar a eficácia da ventilação alveolar e reduzir substancialmente a quantidade de $\mathrm{CO}_{2}$ que permanece nas vias aéreas, ao final da expiração. Os mecanismos pelos quais esse método reduz a $\mathrm{P}_{\mathrm{a}} \mathrm{CO}_{2}$ são: a) o gás insuflado pelo cateter junto à carina dilui o $\mathrm{CO}_{2}$ que permanece no espaço morto, anatômico, na expiração; e b) o fluxo turbulento, gerado pelo ar em alta velocidade, através do cateter, melhora a mistura gasosa na ponta da sonda endotraqueal. A TGI estaria indicada nas situações em que se deseja uma redução da $\mathrm{P}_{\mathrm{a}} \mathrm{CO}_{2}$ (pacientes com DPOC, hipercapnia permissiva) sendo utilizada em conjunto com a ventilação convencional, auxiliando a remoção do $\mathrm{CO}_{2}{ }^{(68)}$.

\subsubsection{Ventilação pulmonar independente}

\section{(ILV - Independent Lung Ventilation)}

Essa abordagem permite a ventilação dos pulmões em separado, através de cânula endotraqueal, com dupla luz e dois ventiladores operando simultaneamente. A ILV está indicada nos casos onde existem importantes diferenças de simetria no grau e extensão do acometimento de cada pulmão. A região mais lesada e, portanto, menos complacente é ventilada com um volume corrente menor que o lado não afetado, evitando-se a hiperinsuflação dessas regiões ${ }^{(57)}$.

\subsubsection{Ventilação volumétrica assistida com pressão suporte (VAPS)}

Teoricamente, a ventilação assistida deveria ser sincronizada com os esforços do paciente. Contudo, isso pode não acontecer, e, como já foi demonstrado na literatura ${ }^{(69,70,71)}$, algumas vezes o trabalho respiratório do paciente pode ser maior nos ciclos assistidos do que na respiração espontânea. Isso acontece porque, ao deflagar o ciclo assistido, o ventilador fornece ao paciente um fluxo de ar constante e fixo, até atingir o volume pré-determinado. Em virtude disso, mesmo tendo um esforço inspiratório elevado, ele não consegue alterar o fluxo programado, havendo uma assincronia entre a velocidade e a força de contração da musculatura diafragmática do paciente e a velocidade com que o ar chega até os alvéolos. Quando a contração do diafragma é muito rápida em relação ao fluxo inspiratório, é como se o paciente estivesse respirando através de uma válvula fechada, acarretando grandes pressões pleurais, negativas. O VAPS é um modo ventilatório, utilizado para minimizar esses problemas $^{(72,73,74)}$ através da utilização da pressão suporte nos ciclos volumétricos assistidos, proporcionando um melhor sincronismo entre o fluxo desejado pelo paciente e o oferecido pelo ventilador, e, por conseguinte, diminuindo o trabalho respiratório do paciente. O VAPS, portanto, é uma modalidade que modifica os padrões de fluxo nos modos Assistido/Controlado e SIMV. Os ciclos respiratórios com VAPS podem ser feitos através de esforço espontâneo (assistido) ou pelo ventilador (controlado) e terminam quando atingem um volume pré-determinado. Costuma-se utilizar o nível de pressão de suporte um pouco abaixo do pico de pressão, obtido na ventilação mecânica convencional. Essa estratégia ventilatória está indicada naqueles pacientes com fadiga muscular, que necessitam de uma redução do trabalho respiratório, permitindo uma melhor "interface" entre o paciente e o ventilador. Cabe ressaltar também que a VAPS atua nos ciclos mandatórios e não nos espontâneos, já que a pressão suporte, nos ciclos espontâneos, tem como finalidade o "desmame" da ventilação mecânica .

\subsubsection{Assistência extracorpórea pulmo-} nar (ELA - Extracorporeal Lung Assist)

É um método de suporte extracorpóreo, usado na tentativa de melhorar a troca gasosa em pacientes com falência respiratória, aguda, grave, refratária. Baseia-se num sistema de trocas gasosas, realizado fora do corpo, e pode ser utilizado em duas técnicas básicas.

\section{a) Oxigenação de Membrana Extracorpórea - ECMO}

$\mathrm{O}$ sangue sai de uma veia central do paciente, passa pelo oxigenador e retorna através de uma artéria, onde o objetivo principal é devolver o sangue já oxigenado para o paciente. Entre 1974 e 1976, o "National Heart Lung and Blood Institute", realizou um estudo multicêntrico, envolvendo noventa (90) pacientes com falência respiratória, severa, comparando ECMO e ventilação convencional, e constatou que a taxa de sobrevida foi semelhante nos dois grupos, de $9,5 \%$ e $8,3 \%$, respectivamente. Esses achados deixaram essa técnica abandonada por longo tempo ${ }^{(75)}$. Atualmente, o seu emprego tem sido reservado para pacientes que estão aguardando o transplante pulmonar. Além disso, a ECMO é uma técnica bastante onerosa, necessita de equipe especializada para sua opera- 
ção e pode ser observada a ocorrência de complicações, como sangramento, embolia gasosa e sepse.

\section{b) Remoção extracorpórea de $\mathrm{CO}_{2}-\mathrm{ECCO}_{2} \mathrm{R}$}

O sistema utilizado é o venovenoso, cujo intuito principal é retirar $\mathrm{CO}_{2}$. O sangue sai de uma veia central e retorna ao átrio direito. Essa técnica está associada à ventilação com pressão positiva e baixa freqüência respiratória. Em determinados casos de SARA, é necessária a redução drástica do volume corrente para evitar barotrauma e volutrauma, cujas repercussões sobre a hipoventilação alveolar, presente são óbvias. Nessas situações, a $\mathrm{ECCO}_{2} \mathrm{R}$ tem um lugar estratégico como técnica coadjuvante, na tentativa de normalização da $\mathrm{P}_{\mathrm{a}} \mathrm{CO}_{2}$.

Gattinoni et al. têm utilizado a técnica denominada ventilação com pressão positiva e baixa frequiência respiratória ${ }^{(52)}$. Utiliza-se frequiência ventilatória de 3 a 5 ciclos/min, níveis adequados de PEEP e pressão limitada a 30-35 $\mathrm{cmH}_{2} \mathrm{O}$. Com essa abordagem, a oxigenação é garantida pelo pulmão recrutado pelo PEEP (oxigenação apnéica) com concomitante remoção extracorpórea do $\mathrm{CO}_{2}$. As complicações dessa modalidade de assistência extracorpórea são similares à ECMO. Devido à ausência de diferenças significativas nas taxas de sobrevida em relação à ventilação mecânica convencional, o método não deve ser utilizado como rotina , mas somente em algumas situações de exceção.

\subsection{8 Óxido nítrico (NO)}

Recentemente, muita atenção tem sido dada ao papel exercido pelo óxido nítrico (NO) na SARA. Em 1987, o NO foi reconhecido como importante vasodilatador da musculatura lisa dos vasos, tendo sido denominado de "endothelium derived relaxing factor EDRF". O NO é sintetizado pelo endotélio vascular a partir da arginina e atua como vasodilatador local. A inalação de óxido nítrico na SARA melhora as trocas gasosas e diminui a pressão na artéria pulmonar, tendo, como resultado, um efeito benéfico sobre a hipoxemia refratária e sobre as desigualdades da relação ventilação/perfusão( ${ }^{(76)}$. No entanto, no estudo extenso de revisão da literatura, versando sobre o uso do NO na SARA, Dinh-Xuan et al. ${ }^{(77)}$ demonstraram que existem muitas dúvidas na concentração de NO a ser inalado, durante o tratamento dessa entidade. A diferença de concentração utilizada pelos diversos autores diferiram muito, não existindo um consenso sobre o real impacto da ventilação mecânica, com enriquecimento do ar inspirado com NO, sobre a mortalidade dos pacientes com SARA.

\section{DESMAME}

O desmame da ventilação pulmonar mecânica deverá ser feito, tão logo o fator etiológico tenha sido removido ou, pelo menos, estabilizado, devendo ser iniciado de maneira gradual. É mandatória a realização de uma avaliação de certas condições gerais ${ }^{(78,79)}$ antes de iniciarmos o desmame, ou seja:

- correção do fator etiológico que levou o paciente ao suporte ventilatório;

- retirada de sedativos (ou doses mínimas) e relaxantes musculares;

- estabilidade hemodinâmica;

- paciente alerta;

- controle de fatores pulmonares como respiração descoordenada, broncoespasmo, infecção e edema;

- correção de distúrbios metabólicos, principalmente os de natureza hidroeletrolítica e ácidobásica.

Uma variedade de métodos para a retirada da ventilação mecânica têm sido propostos, porém, até o momento, não existe um "gold standard" na literatura sobre a melhor alternativa a ser empregada para o desmame ${ }^{(80,81)}$. A escolha do melhor método deverá sempre ser baseada nas características individuais de cada paciente e na experiência da equipe médica. Basicamente, os três métodos mais utilizados na prática diária, em nossa Unidade de Terapia Intensiva, para a retirada dos pacientes da ventilação mecânica, são os seguintes: a) retirada gradual com tubo T; b) ventilação mandatória, intermitente, sincronizada (SIMV) e c) ventilação com suporte de pressão (PSV).

\subsection{Retirada gradual com tubo $\mathbf{T}$}

Esse método permite ao paciente respirar espontaneamente, por um período de tempo pré-determinado, intercalado com a ventilação mecânica, através da conexão de um tubo $\mathrm{T}$, enriquecido com oxigênio, ao tubo endotraqueal do paciente. Inicia-se com períodos de cinco (5) minutos de respiração espontânea, a cada trinta (30) a cento e oitenta (180) minutos, aumentando-se, progressivamente, esse tempo até que o paciente consiga respirar espontaneamente entre trinta (30) e cento e vinte (120) minutos. $\mathrm{O}$ tubo $\mathrm{T}$ proporciona uma resistência menor do que a dos circuitos dos ventiladores, diminuindo o trabalho da musculatura respiratória e, conseqüentemente, melhorando o desempenho e a força de contração muscular. 


\subsection{Ventilação mandatória intermitente sincro- nizada (SIMV)}

Esta técnica possibilita que o paciente respire espontaneamente entre os ciclos controlados pelo ventilador. O modo sincronizado evita que o ciclo mecânico se inicie após a fase inspiratória do paciente, e, por conseguinte, prevenindo os pulmões contra as lesões provocadas pelo barotrauma. Tão logo o paciente se encontre pronto para o desmame, a frequiência da SIMV é diminuída, gradativamente, de 1 a 3 ciclos/min, em cada etapa, com análise dos gases sangüíneos após trinta (30) minutos. Se as condições clinicogasométricas do paciente permitirem, reduz-se a freqüência da SIMV até 6 ciclos/min ou menos, e, então, procede-se a extubação. Um inconveniente importante dessa modalidade de desmame é o aumento da resistência, causado pelo circuito do ventilador, o que provoca um aumento do trabalho respiratório e fadiga muscular.

\subsection{Ventilação com suporte de pres- são (PSV)}

Essa técnica foi introduzida na tentativa de diminuir o trabalho respiratório do paciente, ocasionado pela resistência gerada pelos circuitos dos ventiladores, cânula endotraqueal e válvulas de demanda dos ventiladores. Após o paciente deflagar a abertura da válvula, uma pressão positiva, pré-estabelecida pelo médico assistente, é aplicada ao circuito, facilitando a sua respiração. O próprio paciente controla o tempo inspiratório, a frequiência e a taxa de fluxo inspiratório. Os valores empregados na PSV são empíricos variando entre 5 e $15 \mathrm{cmH}_{2} \mathrm{O}$ e a retirada é feita gradualmente, com decréscimos de 3 a $6 \mathrm{cmH}_{2} \mathrm{O}$, até um valor mínimo de PSV, em torno de $5 \mathrm{cmH}_{2} \mathrm{O}^{(82)}$. Uma das vantagens dessa técnica, além de diminuir o trabalho respiratório e, conseqüentemente, prevenir a fadiga da musculatura respiratória, é um melhor "conforto" e sincronismo entre o paciente e o ventilador .
Com base no que foi relatado acima e nos dados da literatura, elaboramos, na Unidade de Terapia Intensiva da Unidade de Emergência do Hospital das Clínicas - FMRPUSP, um protocolo de desmame para pacientes submetidos à ventilação mecânica (Figura 3).

\section{Problema de base resolvido}

2. pH normal

\section{Sedação mínima}

4. Ventilação Mecânica Mínima PEEP(CPAP) $<5 \mathrm{~cm} \mathrm{H}_{2} \mathrm{O}$

SIMV $<6 / \mathrm{min}$

$\mathrm{PaO}_{2}>60 \mathrm{mmHg}\left(\mathrm{F}_{1} \mathrm{O}_{2}<0.4\right)$

5. Sinais Vitais/Hemodinâmicos

Freqüência cardíaca $<120$ bpm

Freqüência respiratória $(\mathrm{FR})<30$ inc./min

Pressão arterial média $>65 \mathrm{mmHg}$

Temperatura corpórea entre 36 e $38.5^{\circ} \mathrm{C}$

Volume corrente $\left(\mathrm{V}_{\mathrm{T}}\right)>5 \mathrm{ml} / \mathrm{kg}$

Esforço inspiratório negativo $>-20 \mathrm{~cm} \mathrm{H}_{2} \mathrm{O}$

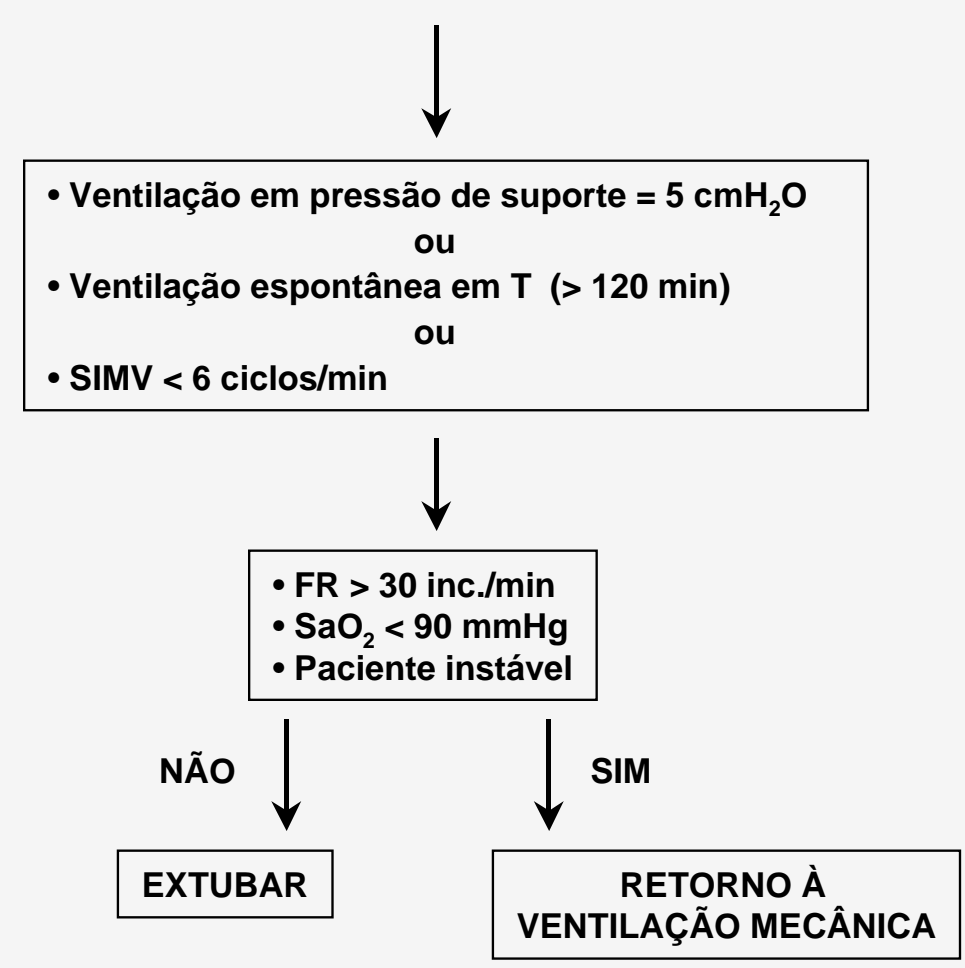

Figura 3 - Proposição de protocolo de "desmame" da ventilação mecânica. 


\section{CONCLUSÕES}

A despeito de todo o avanço tecnológico, da nova geração de ventiladores microprocessados, da monitorização respiratória sofisticada e da utilização, em testes, de diversas drogas, na terapia da SARA, a verdade é que existem muitas dúvidas, até o presente momento, sobre todos os aspectos envolvidos nesta síndrome. A superinfecção pulmonar, com possibilidade de sepse, permanece ainda um grande pro- blema, contribuindo, substancialmente, para a estabilidade nos índices de mortalidade da SARA. É possível que, com uma identificação mais precoce dos fatores de risco para o desenvolvimento da síndrome, o suporte metabólico, imediato, uma melhor e mais agressiva ressuscitação dos estados de choque, o uso de óxido nítrico inalatório e alterações na abordagem ventilatória possam, talvez, no futuro, permitir uma evolução menos desfavorável dessa complexa patologia.

ANTONIAZZI P et al. Acute respiratory distress syndrome (ARDS). Medicina, Ribeirão Preto, 31: 493-506, oct./dec. 1998.

ABSTRACT: The acute respiratory distress syndrome (ARDS) is described as an acute lung injury, with pulmonary edema and severe hypoxemia, associated with high mortality rates (10$90 \%$, mean $=50 \%$ ), depending upon its etiology. Although ARDS had been described for 30 years, its several aspects such as definition, physiopathology and adequate treatment still remain unclear.

The purpose of this paper is to describe an updated review of the clinical, radiologic and physiopathological aspects of ARDS, in order to enhance the knowledge on this syndrome and to provide the guidelines of the therapeutics, and mechanical ventilation techniques used to assess these patients.

UNITERMS: Respiratory Insufficiency. Anoxemia. Respiratory Distress Syndrome, Adult.

\section{REFERÊNCIAS BIBLIOGRÁFICAS}

1 - ASHBAUGH DG et al. Acute respiratory distress in adults. Lancet 2: 319-323, 1967.

2 - PETTY TL \& ASBAUGH DG. The adult respiratory distress syndrome: clinical features, factors influencing prognosis and principles of management. Chest 60: 233-239,1971.

3 - SLOANE PJ et al. A multicenter registry of patients with acute respiratory distress syndrome: Physiology and outcome. Am Rev Respir Dis 146: 419-426, 1992.

4 - FOWLER AA ey al. Adult respiratory distress syndrome: Risk with common predispositions. Ann Intern Med 98: 593-597, 1983

5 - PEPE PE et al. Clinical predictors of the adult respiratory distress syndrome. Am J Surg 144: 124-130, 1982.

6 - MURRAY JF et al. An expanded definition of the adult respiratory distress syndrome. Am Rev Respir Dis 138: 720723, 1988.

7 - MARC $M$ et al. Establishing the relative accuracy of three new definitions of the adult respiratory distress syndrome. Crit Care Med 23: 1629-1637, 1995.

8 - KNAUS WA et al. Evaluation of definitions for adult respiratory distress syndrome. Am J Respir Crit Care Med 150: 311-317, 1994.
9 - MONTGOMERY AB et al. Causes of mortality in patients with the adult respiratory distress syndrome. Am Rev Respir Dis 132: 485-489, 1985.

10 - BERNARD GR et al. High-dose corticosteroids in patients with the adult respiratory distress syndrome. $\mathbf{N}$ Engl J Med 317: 1565-1570, 1987.

11 - LEONARD DH et al. Clinical risks for developement of the acute respiratory distress syndrome. Am J Respir Crit Care Med 151: 293-301, 1995.

12 - RAMONA LD et al. Identification of patients with acute lung injury: Predictors of mortality. Am J Respir Crit Care Med 152: 1818-1824, 1995.

13 - MEDURI GU et al. Fibroproliferative phase of ARDS: Clinical findings and effects of corticosteroids. Chest 100: 943952, 1991.

14 - SUTER PM et al. $\mathrm{N}$-acetylcysteine enhances recovery from acute lung injury in man. Chest 105: 190-194, 1994.

15 - DOYLE HR et al. Adult respiratory distress syndrome (ARDS) and end-stage liver disease: Resolution following liver transplantation. Am Rev Respir Dis 145: A 319, 1992 Abstract.

16 - McHUGH LG et al. Recovery of function in survivors of the acute respiratory distress syndrome. Am J Respir Crit Care Med 150: 90-94, 1994. 
17 - MATTHAY MA. The adult respiratory distress syndrome: Definition and prognosis. Clin Chest Med 11: 575-580, 1990.

18 - BERNARD GR et al. Report of the American-European consensus conference on ARDS: definitions, mechanisms, relevant outcomes and clinical trial coordination. Intens Care Med 20: 225-232, 1994.

19 - BASILE FILHO A \& CAPONE NETO A. Síndrome da angústia respiratória do adulto. Rev Bras Anest 38: 431-443, 1988.

20 - MATTHAY MA. Síndrome da angústia respiratória do adulto: Definição e prognóstico. Clín Doenças Pulm 4: 557-562, 1990.

21 - ABELDA SM \& BUCK CA. Integrins and the other cell adhesion molecules. FASEB (Experimental Biology) 4: 2868-2880, 1990.

22 - MOORE KL et al. Endotoxin enhance tissue factor and supresses thrombomodulin expression of human vascular endothelium in vitro. J Clin Invest 79: 124-130, 1987.

23 - ERTEL W et al. The complex pattern of cytokines in sepsis. Association between prostaglandins, cachectin and interleukins. Ann Surg 214: 141-148, 1991

24 - KATZENSTEIN AA et al. Diffuse alveolar damage: The role of oxygen, shoch and related factors. Am J Pathol 85: 210-222, 1976.

25 - TEPLITZ C. The core pathobiology and integrated medical science adult acute respiratory insufficiency. Surg Clin North Am 56: 1091-1133, 1976.

26 - FUKUDA $Y$ et al. The role of intraalveolar fibrosis in the process of pulmonary structural remodeling in patients with diffuse alveolar damage. Am J Pathol 126: 171-182, 1987.

27 - PISON U et al. Surfactant abnormalities in patients with respiratory failure after multiple trauma. Am Rev Respir Dis 140: 1033-1039, 1989.

28 - CHURG A et al. Broncho pulmonary dysplasia in the adult. Am Rev Respir Dis 127: 117-120, 1983.

29 - ARTIGAS A et al. Long-term follow-up of pulmonary function recovery after adult respiratory distress syndrome. Intens Care Med 6: 26, 1980. Suppl.

30 - ARMSTRONG BW \& MACINTYRE NR. Pressure-controlled inverse ratio ventilation that avoids air trapping in the adult respiratory distress syndrome. Crit Care Med 23: 279285, 1995.

31 - CARVALHO CR \& AMATO MB. Tratamento da síndrome do desconforto respiratório do adulto. J Pneumol 16: 212228, 1990.

32 - SCHUMACKER PT \& CAIN SM. The concept of a critical oxygen delivery. Intens Care Med 13: 223-229, 1987.

33 - DORINSKY PM \& GADEK JE. Mechanisms of multiple nonpulmonary organ failure in ARDS. Chest 96: 885-892, 1989.
34 - NELSON LD. Mixed venous oxymetry. In: SNYDER JV \& PINSKY MR. Oxygen transport in the critically ill. Year Book Medical. Chicago, London, p. 235-248, 1987.

35 - SNYDER JV. The developement of supported ventilation: a critical summary. In: SNYDER JV \& PINSKY MR. Oxigen transport in the critically ill. Year Book Medical. Chicago, London, p. 283-294, 1987.

36 - SHUMER W. Steroids in the treatment of clinical septic shock. Ann Surg 184: 333-339, 1976.

37 - SALDIVA PH et al. Changes in the pulmonary glicocorticoid receptor content in the course of intersticial disease. Chest 88: 417-419, 1985.

38 - BONE RC et al. Early methylprednisolone treatment for septic syndrome and the adult respiratory distress syndrome. Chest 92: 1032-1036, 1987.

39 - BERNARD GR et al. High-dose corticosteroids in patients with the adult respiratory distress syndrome. $\mathbf{N}$ Engl J Med 317: 1565-1570, 1987.

40 - LUCE JM et al. Ineffectiveness of high-dose methylprednisolone in preventing parenchymal lung injury and improving mortality in patients with septic shock. Am Rev Respir Dis 138: 62-68, 1988.

41 - ASHBAUGH DG \& MAIER RV. Idiopathic pulmonary fibrosis in adult respiratory distress syndrome. Arch Surg 120: 530535,1985

42 - HOOPER RG \& KEARL RA. Established ARDS treated with a sustained course of adrenocortical steroids. Chest 97: 138143, 1990.

43 - MEDURI GU. Fever in late adult respiratory distress syndrome: etiology, pathophysiology and diagnostic evaluation. Semin Respir Crit Care Med 4: 308-324, 1994.

44 - GREGORY TJ et al. Survanta supplementation in patients with acute respiratory distress syndrome. Am J Respir Crit Care Med 149: A 567, 1994 Abstract.

45 - BONE RC et al. Randomized double-blind, multicenter study of prostaglandin $E_{1}$ in patients with the adult respiratory distress syndrome. Chest 96: 114-119, 1989.

46 - JEPSEN S et al. Antioxidant treatment with $\mathrm{N}$-acetylcysteine during adult respiratory distress syndrome: A prospective, randomized, placebo-controlled study. Crit Care Med 20: 918-923, 1992.

47 - PEPE PE et al. Clinical predictors of the adult respiratory distress syndrome. Am J Surg 144: 124-129,1982.

48 - TSUNO $\mathrm{K}$ et al. Acute lung injury from mechanical ventilation at moderately high airway pressures. J Appl Physiol 69: 956-961, 1990.

49 - DREYFUSS D et al. Mechanical ventilation induced pulmonary edema. Am J Respir Crit Care Med 151: 1568-1575, 1995.

50 - AMATO MB et al. Respiratory failure due to alveolar hemorrhage: diagnostic troubles and the impact of a new ventilatory strategy. Am Rev Respir Dis 145: A 75, 1992 Abstract. 
51 - DREYFUS D \& SAUMON G. Barotrauma is volutrauma, but wich volume is the one responsible? Intens Care Med 18: 139-141, 1992.

52 - GATTINONI L et al. Low-frequency positive-pressure ventilation with extra-corporeal $\mathrm{CO}_{2}$ removal in severe acute respiratory failure. JAMA 256: 881-886, 1986.

53 - HICKLING KG et al. Low mortality associated with low volume pressure limited ventilation with permissive hypercapnia in severe adult respiratory distress syndrome. Intens Care Med 16: 372-377, 1990.

54 - MARCY TW \& MARINI JJ. Inverse ratio ventilation in ARDS: Rationale and implementation. Chest 100: 494-504, 1991.

55 - AMATO MB et al. Benefical effects of the "open lung approach"with low distending pressures in ARDS: A prospective randomized study on mechanical ventilation. Am J Respir Crit Care Med 152: 1835-1846, 1995.

56 - GATTINONI et al. Body position changes redistribute lung computed tomographic density in patients with acute respiratory failure. Anesthesiology 74: 15-23, 1991.

57 - BAEHRENDTZ S \& HEDENSTIERNA G. Differencial ventilation and seletive positive end-expiratory pressure: Effects on patients with acute bilateral lung disease. Anesthesiology 61: $511-517,1984$.

58 - NAHUM A et al. Efficacy of expiratory tracheal gas insufflation in a canine model of lung injury. Am $\mathbf{J}$ Respir Crit Care Med 152: 482-495, 1995.

59 - KACMARECK RM \& MEKLAUS GJ. The new generation of mechanical ventilators. Crit Care Med 3: 551-578, 1990.

60 - MARINI JJ. Patient-ventilator interation: rational strategies for acute ventilatory management. Respir Care 38: 482493, 1993.

61 - SLUTSKY AS. Mechanical ventilation. Chest 104: 18331859, 1993.

62. KACMAREK RM \& HICKLING KG. Permissive hypercapnia. Respir Care 38: 373-387, 1993.

63 - FEIHL F \& PERRET C. Permissive hypercapnia. How permissive should we be? Am J Respir Crit Care 150: 17221737, 1994.

64 - HICKLING KG et al. Low mortality rate in adult respiratory distress syndrome using low volume, pressure-limited ventilation with permissive hypercapnia: A prospective study. Crit Care Med 22: 1568-1578, 1994.

65 - GENTILLELO LM et al. Permissive hypercapnia in trauma patients. J Trauma 39: 846-853, 1995.

66 - AMATO MB et al. Improved survival in ARDS: Benefical effects of lung protective strategy. Am J Respir Crit Care Med 153: A531, 1996. Abstract.
67 - CARLON CG et al. High-frequency jet ventilation: A prospective randomised evaluation. Chest 84: 551-559, 1983.

68 - RAVENSCRAFT SA et al. Intratracheal gas insuflation augments alveolar ventilation during volume cycled mechanical ventilation in patients. Am Rev Respir Dis 145: A 529, 1992. Abstract.

69 - MACINTYRE NR. Weaning from mechanical ventilatory support: volume assisting intermittent breaths versus pressure assisting breath. Resp Care 33: 121-125, 1988.

70 - MARINI JJ. Strategies to minimize breathing effort during mechanical ventilation. Crit Care Clin 6: 635-661, 1990.

71 - MARINI JJ et al. Bedside estimation of the inspiratory work of breathing during mechanical ventilation. Chest 89: 5663, 1986.

72 - AMATO MB et al. Volume assisted pressure support ventilation: A clinical study. Resp Care 35: 1113, 1990. Abstract.

73 - AMATO MB et al. Volume assured pressure support ventilation (VAPSV). A new approach for reducing muscle workload during acute respiratory failure. Chest 102: 1225-1234, 1992.

74 - BONASSA $\mathrm{J}$ et al. Volume assisted pressure support ventilation. Resp Care 35: 1113, 1990. Abstract.

75 - ZAPOL WM et al. Extracorporeal membrane oxigenation in severe acute respiratory failure. JAMA 242: 2193-2196, 1979.

76 - ROSSAINT R et al. Inhaled nitric oxide for the adult respiratory distress syndrome. N Engl J Med 328: 399-405, 1993.

77 - DINH-XUAN AT et al. The use and misuse of exogenous endothelial-derived vasodilators in acute respiratory failure. Intens Care Med 23: 1110-1118, 1997.

78 - SPORN PH \& MORGANROTH ML. Discontinuation of mechanical ventilation. Clin Chest Med 9: 113-126, 1988.

79. LESSARD MR \& BROCHARD LJ. Weaning from ventilatory support. Clin Chest Med 17: 475-489, 1996.

80. BROCHARD LJ et al. Comparison of three methods of gradual withdrawal from ventilatory support during weaning from mechanical ventilation. Am J Respir Crit Care Med 150: 896-903, 1994.

81. ESTEBAN A et al. A comparison of four methods of weaning patients from mechanical ventilation. $\mathbf{N}$ Engl $\mathbf{J}$ Med 332: 345-350, 1995.

82. TOBIN MJ \& YANG K. Weaning from mechanical ventilation. Crit Care Clin 6: 725-747, 1990.

Recebido para publicação em 04/03/98

Aprovado para publicação em 07/10/98 